\title{
Is there a clinically significant seasonal component to hospital admissions for atrial fibrillation? Ross EG Upshur*1,2,3, Rahim Moineddin ${ }^{2}$, Eric J Crighton ${ }^{1}$ and Muhammad Mamdani 4,5,6
}

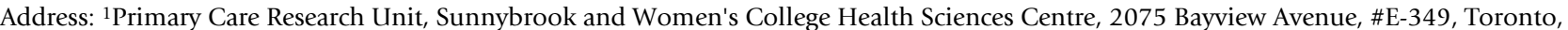
Ontario, Canada M4N 3M5, 2Departments of Family and Community Medicine, University of Toronto, 256 McCaul, Street, 2nd Floor, Toronto, Ontario, Canada M5T 2W5, ${ }^{3}$ Department of Public Health Sciences, University of Toronto, McMurrich Building, 12, Queen's Park Crescent W., Toronto, Ontario, Canada M5S 1A8, ${ }^{4}$ Institute of Clinical Evaluative Sciences (ICES), 2075 Bayview Avenue, Toronto, Ontario, Canada M4N 3M5, ${ }^{5}$ Health Policy Management and Evaluation, University of Toronto, McMurrich Building, 2nd Floor, 12 Queen's Park Crescent W., Toronto, Ontario, Canada M5S 1A8 and 'Faculty of Pharmacy, University of Toronto, 19 Russell Street, Toronto, Ontario, Canada, M5S 2S2
\end{abstract}

Email: Ross EG Upshur* - rupshur@idirect.com; Rahim Moineddin - rahim.moineddin@utoronto.ca; Eric J Crighton - eric.crighton@sw.on; Muhammad Mamdani - muhammad.mamdani@ices.on.ca

* Corresponding author

Published: 19 March 2004

BMC Health Services Research 2004, 4:5
Received: 29 October 2003

Accepted: 19 March 2004

This article is available from: http://www.biomedcentral.com/I472-6963/4/5

(C) 2004 Upshur et al; licensee BioMed Central Ltd. This is an Open Access article: verbatim copying and redistribution of this article are permitted in all media for any purpose, provided this notice is preserved along with the article's original URL.

\begin{abstract}
Background: Atrial fibrillation is a common cardiac dysrhythmia, particularly in the elderly. Recent studies have indicated a statistically significant seasonal component to atrial fibrillation hospitalizations.

Methods: We conducted a retrospective population cohort study using time series analysis to evaluate seasonal patterns of atrial fibrillation hospitalizations for the province of Ontario for the years 1988 to $200 \mathrm{I}$. Five different series methods were used to analyze the data, including spectral analysis, XII, R-Squared, autocorrelation function and monthly aggregation.

Results: This study found evidence of weak seasonality, most apparent at aggregate levels including both ages and sexes. There was dramatic increase in hospitalizations for atrial fibrillation over the years studied and an age dependent increase in rates per 100,000. Overall, the magnitude of seasonal difference between peak and trough months is in the order of I.4 admissions per 100,000 population. The peaks for hospitalizations were predominantly in April, and the troughs in August.

Conclusions: Our study confirms statistical evidence of seasonality for atrial fibrillation hospitalizations. This effect is small in absolute terms and likely not significant for policy or etiological research purposes.
\end{abstract}

\section{Background}

Atrial fibrillation is the most common cardiac arrhythmia in the elderly population requiring medical treatment. The prevalence of this disease is clearly related to age and can be as high as 15 to $18 \%$ after the age of $80[1,2]$. The seasonality of hospitalizations for atrial fibrillation has been the focus of epidemiological study as seasonality is a potential clue to etiology. Recent studies examining the 
seasonality of atrial fibrillation using monthly aggregations of emergency reports over a 10 -year period in one study, and emergency room visits over a 1 -year period in another, both found statistically significant seasonal differences in monthly values, with peaks typically occurring in the winter and troughs in the summer $[3,4]$. Frost et al in a study of hospitalizations for atrial fibrillation in Denmark found a winter peak and summer trough, with a small but statistically significant relative risk of 1.20 (95\% confidence interval: $1.12,1.29$ ) for winter events [5]. They also reported an inverse relationship between mean outdoor temperature and atrial fibrillation.

Although significant seasonality was reported in each of the above studies, they are somewhat limited methodologically in that events were aggregated into monthly categories, rates were not reported, and trends over time were not examined. We believe this type of analysis obscures important elements of year to year variability, the differential effects of age and sex, as well as the absolute magnitude of the seasonal effect. These elements are essential in determining whether statistically determined seasonality has etiologic or policy relevance. To address the above limitations and to better understand the seasonality of atrial fibrillation hospitalizations, we analyzed the seasonality of atrial fibrillation hospitalization rates in the Ontario population by age and gender. We employed different approaches to seasonality detection: spectral analysis, autocorrelation function, and the X11 approach, $R_{\text {Autoreg }}^{2}$ in addition to monthly aggregation in order to study trends and season to season variability.

\section{Methods}

We conducted a retrospective population-based study to assess temporal patterns in all hospitalizations for atrial fibrillation for the population 40 years of age and older from April 1, 1988 to March 31, 2001. Approximately 14 million residents of Ontario, Canada eligible for universal health care coverage during this time were included for analysis. The database used was the Canadian Institute for Health Information Discharge Abstract Database which records discharges from all in patient hospital stays in Ontario acute care hospitals. All records with a primary discharge diagnosis of atrial fibrillation (ICD-9 code: 427.3) were selected. This indicates that atrial fibrillation was the primary reason for the hospitalization. The numerator consisted of the total number of hospitalizations for each month. Denominators were constructed from annual census data provided by Statistics Canada for each age group for residents of Ontario. Monthly population estimates were derived through linear interpolation. From this data, admission rates per 100,000 population, normalized for length of month, were calculated. We excluded all transfers from within 1 acute care hospital to another within this study group. We constructed time series for overall hospitalizations, overall by gender and by 10 year age bands and gender. Time series analysis was conducted to assess seasonal variations and trends over time and to account for autocorrelation, which is typically problematic with time-related data. Five methods were used to test for seasonality: spectral analysis, X11, autocorrelation, monthly aggregation and $R_{\text {Autoreg }}^{2}$.

1) Spectral analysis: Spectral analysis is a useful frequency domain tool for detecting the existence of periodicity in a time series. This can be achieved by plotting the periodogram or spectral density of the series against either period or the frequency. There are 2 statistical tests for testing the periodicity of the series: The Fisher's Kappa test and the Bartlett's Kolmogorov-Smirnov (BKS) test. Fisher's Kappa tests the null hypothesis that the series is Gaussian white noise against the alternative hypothesis that the series contains an added deterministic periodic component of unspecified frequency. The BKS test compares the normalized cumulative periodogram with the cumulative distribution function of the uniform $(0,1)$ to test the null hypothesis that the series is white noise [6,7].

2) The X11 procedure: 2 tests using this time domain approach were performed, the stable seasonality test and moving seasonality test $[8,9]$. The stable seasonality is a 1 way analysis of variance on the de-trended series with months as the factor. The moving seasonality test is a 2way analysis of variance with month and year as factors.

3) Autocorrelation function: this measures the correlation between observations at different time lags [10].

4) Monthly aggregation.

5) $R_{\text {Autoreg }}^{2}$ which measures the strength of seasonality in a time series. Values of 0 to less than 0.4 represent nonexistent to weak seasonality, 0.4 to less than 0.7 represent moderate to strong seasonality, and 0.7 to 1 represent strong to perfect seasonality.

The data was subjected to logarithmic transformation to stabilize the variance and make the seasonal effect additive [11].

\section{Results}

Table 1 shows the age and sex breakdown for the 13-year cohort. Overall, there were 90,200 hospitalizations, 45,478 for females and 44,722 for males. Figures 1 and 2 show the monthly rates of hospitalizations per 100,000 population, overall, and by age. There is a conspicuous upward trend in rates over the first 4 years overall (Figure 1 ), particularly in the oldest age groups (Figure 2) where 
Table I: Total number of atrial fibrillation hospitalizations by age and gender between the years I988 and 200 I

\begin{tabular}{cccc}
\hline Age group (years) & Male & Female & Both genders \\
$40-49$ & 3890 & 1365 & 5255 \\
$50-59$ & 7518 & 3603 & 11121 \\
$60-69$ & 13253 & 9931 & 23184 \\
$70-79$ & 13851 & 17209 & 31060 \\
$80+$ & 6210 & 13370 & 19580 \\
All ages & 44722 & 45478 & 90200 \\
\hline
\end{tabular}

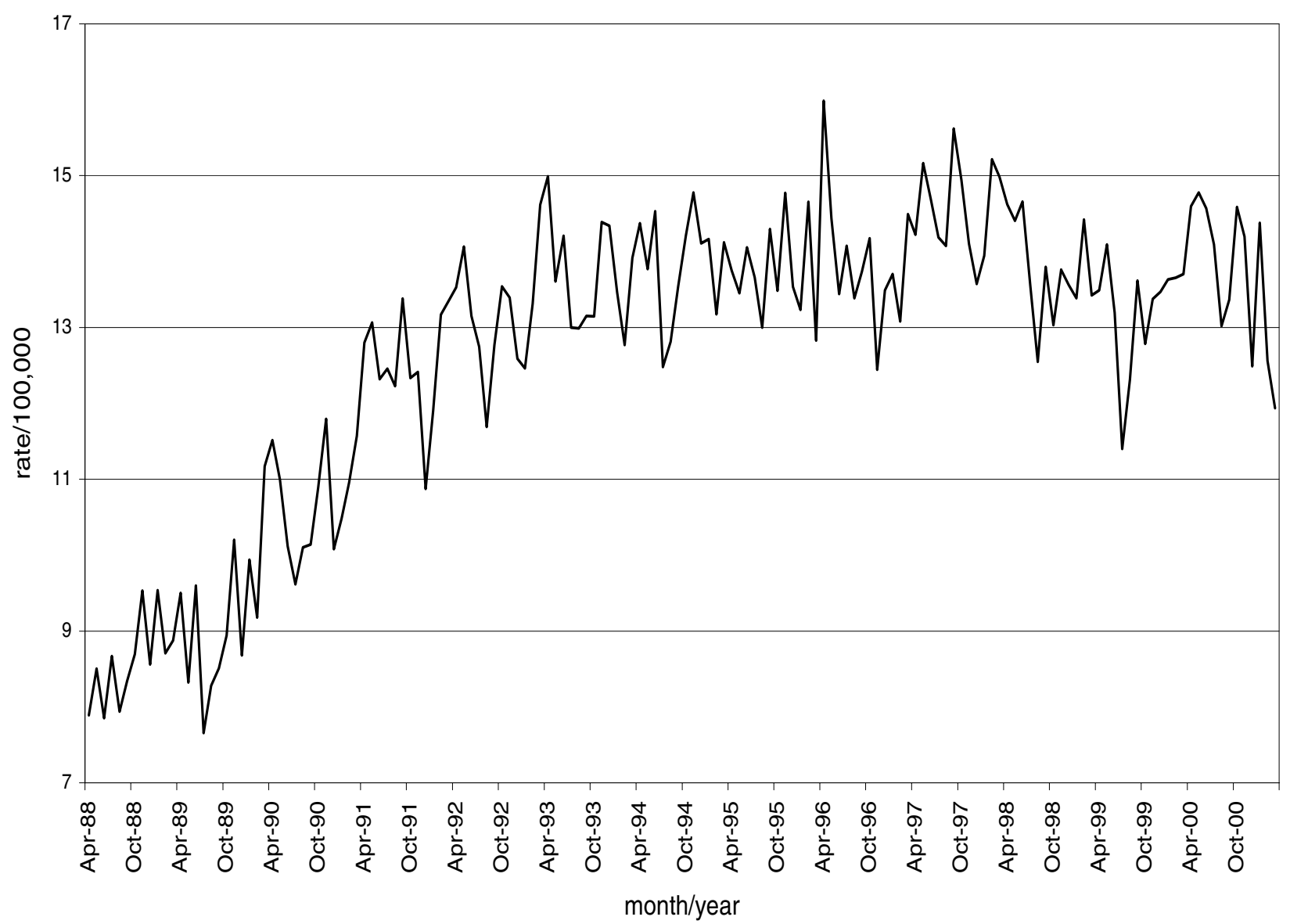

Figure I

Time plot of atrial fibrillation hospitalizations aggregated by all ages and genders expressed as rates per 100,000 .

the rate per 100,000 population doubles during this time. There is a clear relationship between age and hospitalization rates. The rates increase steadily, with average rates of 2 per 100,000 in the 40-49 age group to 50 per 100,000 in the $80+$ age group. There is little difference in rates between males and females except in the younger cohorts (40-49 and 50-59) whereby males have higher hospitalization rates than females. By age 60 the rates are similar between the sexes (data not shown). 


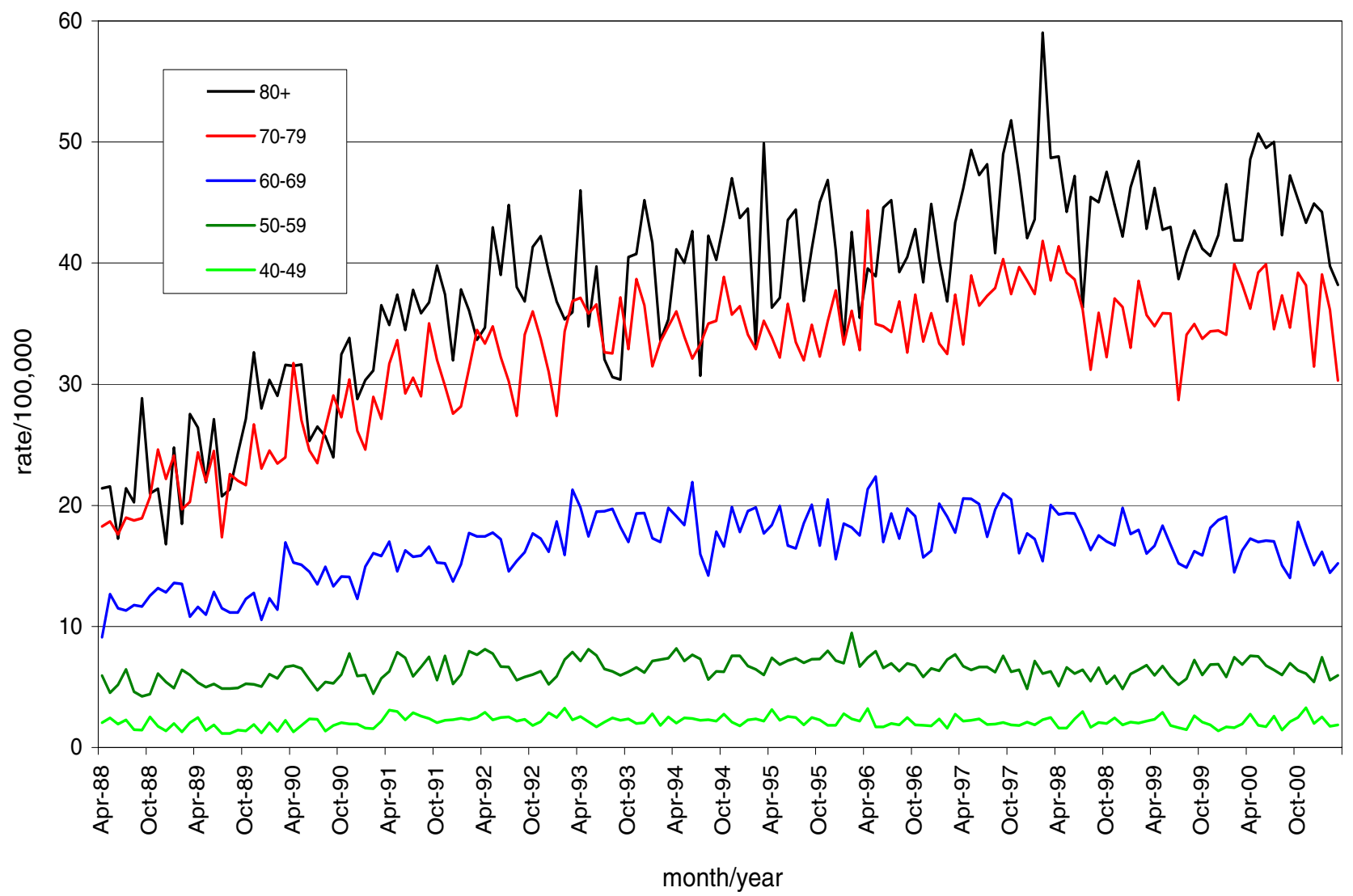

Figure 2

Time plot of atrial fibrillation hospitalizations by age aggregated by genders, expressed as rates per 100,000 .

Table 2 shows the results of the spectral analysis. Statistically significant seasonality is detected by both the Fisher's Kappa and the BKS statistic for both sexes and all ages combined, as well as for females, all ages combined. Within the age and sex analysis there is variability according to age, sex and test.

Table 3 shows the results of the X11 analysis. The age aggregated analysis reveals that in the combined test, there is statistically significant seasonality for males, females and both genders. Within the age and sex analysis there is variability according to age, sex and test.

The autocorrelation function analysis (Table 4) shows significant seasonality for the total population, and no seasonality for men and women. For the total population autocorrelation occurs in a consistent 12 month cycle.
Figure 3 shows the monthly aggregated rates overall and for both genders. There is a conspicuous trough in August. The magnitude of the difference between peak and trough is of the order of 1.3 admissions per 100,000 population, with a small peak in April.

Table 5 summarizes the results of the $R_{\text {Autoreg seasonality }}^{2}$ tests by age and gender. The overall series shows weak seasonality. All other analysis show evidence of weak seasonality.

\section{Discussion}

The results of this analysis confirm a weak seasonal effect for atrial fibrillation hospitalizations in the Ontario population. As the time series analyses indicate, this seasonality is most apparent in aggregate, occurs as a peak effect in spring and largely disappears when age groups and sexes are considered separately. The results indicate a 
Table 2: Results of the spectral analysis testing the seasonality of atrial fibrillation hospitalizations by age and gender between the years 1988 and 2001

\begin{tabular}{|c|c|c|c|c|c|c|}
\hline \multirow[b]{2}{*}{ Age group } & \multicolumn{2}{|c|}{ Male } & \multicolumn{2}{|c|}{ Female } & \multicolumn{2}{|c|}{ Both genders } \\
\hline & $\mathrm{FK}^{\dagger}$ & BKS $\ddagger$ & FK & BKS & FK & BKS \\
\hline $40-49$ & 5.48 & 0.08 & $6.83^{*}$ & 0.12 & 4.82 & 0.12 \\
\hline $50-59$ & 5.77 & $0.18^{*}$ & 4.46 & 0.09 & $7.77^{* *}$ & $0.24 * * *$ \\
\hline $60-69$ & 5.34 & $0.18^{*}$ & 5.97 & 0.08 & $6.52^{*}$ & $0.22^{* *}$ \\
\hline $70-79$ & 4.53 & 0.08 & $7.65 * *$ & 0.12 & $9.35 * *$ & $0.18^{*}$ \\
\hline $80+$ & 4.69 & $0.16^{*}$ & $6.64 *$ & $0.20 * *$ & $9.17 * *$ & $0.23 * * *$ \\
\hline All ages & 5.68 & $0.19 * *$ & $9.20 * *$ & $0.16 *$ & $11.32^{* *}$ & $0.28 * * *$ \\
\hline
\end{tabular}

† FK (Fisher Kappa Test) tests the null hypothesis that the series is Gaussian white noise against the alternative hypothesis that the series contains an added deterministic periodic component of unspecified frequency. $¥$ BKS (Bartelette's Kolmogorov-Smirnov Test) tests the null hypothesis that the series is white noise. $* p<0.05, * * p<0.01$, $* * * p<0.001$

Table 3: Results of the X-I I analysis testing the seasonality of atrial fibrillation hospitalizations by age and gender between the years 1988 and 2001

\begin{tabular}{|c|c|c|c|c|c|c|c|c|c|}
\hline \multirow[b]{2}{*}{ Age group } & \multicolumn{3}{|c|}{ Male } & \multicolumn{3}{|c|}{ Female } & \multicolumn{3}{|c|}{ Both genders } \\
\hline & Stablet & Moving $\ddagger$ & Combined§ & Stable & Moving & Combined & Stable & Moving & Combined \\
\hline $40-49$ & $2.6 I^{* *}$ & 1.52 & not present & 1.15 & 1.42 & not present & 3.20 & 1.12 & not present \\
\hline $50-59$ & $3.16 * * *$ & 0.87 & not present & $2.02 *$ & 1.28 & not present & 4.10*** & 1.79 & present \\
\hline $60-69$ & 1.61 & 0.92 & not present & 0.78 & 0.44 & not present & $2.3 I^{*}$ & 0.63 & not present \\
\hline $70-79$ & 1.48 & 0.78 & not present & $4.08 * * *$ & I.88* & present & $3.95^{* * *}$ & 1.13 & present \\
\hline $80+$ & 1.25 & 1.07 & not present & $2.02 *$ & $3.05^{* *}$ & not present & $2.17^{*}$ & $2.33 *$ & not present \\
\hline All ages & $4.56 * * *$ & 1.43 & present & $4.37 * * *$ & $2.02 *$ & present & 7.16 *** & 1.35 & present \\
\hline
\end{tabular}

† stable seasonality is a one-way analysis of variance on the de-trended series with months as the factor $\ddagger$ moving seasonality test is a two-way analysis of variance with month and year as factors $\S$ combined test $* p<0.05$, $*_{p}<<0.01$, ***p $<0.001$

Table 4: Results of the autocorrelation function (lag I 2) for testing the seasonality of atrial fibrillation hospitalizations by age and gender between the years 1988 and $200 I$

\begin{tabular}{cccc}
\hline Age group (years) & Male & Female & Both genders \\
\hline $40-49$ & 0.0860 & -0.0757 & 0.0578 \\
$50-59$ & 0.1318 & 0.1467 & $0.2246^{*}$ \\
$60-69$ & 0.1089 & 0.1082 & 0.1605 \\
$70-79$ & 0.0484 & 0.1318 & $0.1891^{*}$ \\
$80+$ & 0.0175 & 0.1025 & 0.1543 \\
All ages & 0.1458 & 0.0923 & $0.2179 *$ \\
\hline
\end{tabular}

$*_{\mathrm{p}}<0.05$

conspicuous upward trend for hospitalizations in the late 80 s and early 90 s that has since stabilized.

The strengths of this study are the large sample size, duration of analysis and the use of different methods of analyzing seasonality. Each of the methods in this analysis has different strengths and weaknesses, but we regard the results as mutually supporting despite small disagreements. The overall strength of effect is well captured by the 


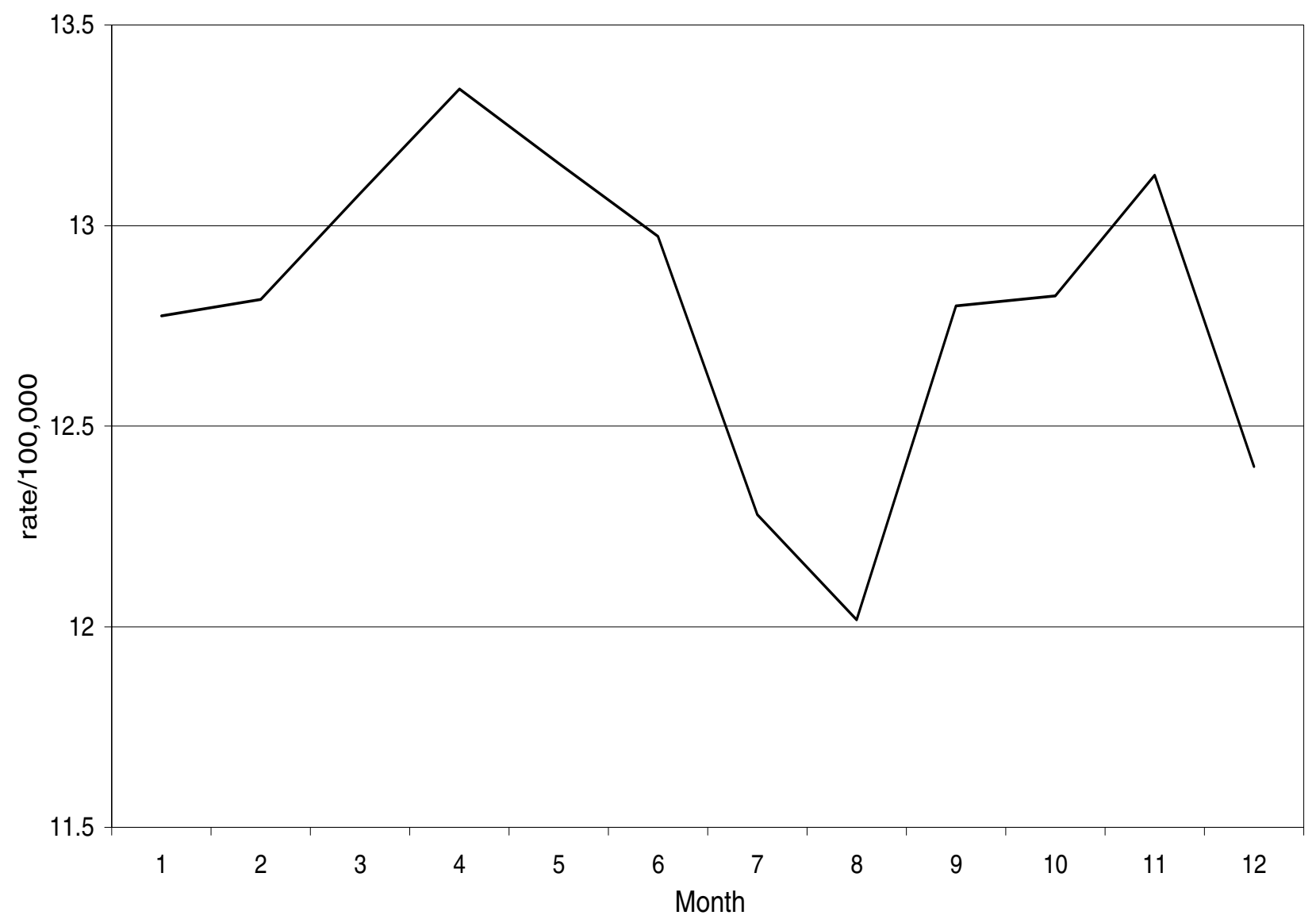

Figure 3

Monthly aggregation of atrial fibrillation hospitalizations expressed as rates per 100,000 .

Table 5: Results of the R-squared autoregression for testing the strength of seasonality of atrial fibrillation hospitalizations by age and gender between the years 1988 and 2001

\begin{tabular}{cccc}
\hline Age group (years) & Male & Female & Both genders \\
\hline $40-49$ & 0.16 & 0.07 & 0.19 \\
$50-59$ & 0.16 & 0.12 & 0.20 \\
$60-69$ & 0.10 & 0.06 & 0.12 \\
$70-79$ & 0.08 & 0.16 & 0.21 \\
$80+$ & 0.07 & 0.13 & 0.11 \\
All ages & 0.21 & 0.28 & 0.30 \\
\hline
\end{tabular}

$R_{\text {Autoreg }}^{2}$ analysis (Table 5). The presence of seasonality is best supported by the analysis that aggregates all admissions. This would indicate that the seasonal effect is apparent at a population level.
The study has several limitations. Firstly, the data base cannot distinguish new onset from chronic atrial fibrillation. However, the purpose of the study was not to calculate seasonal incidence of atrial fibrillation. Secondly, the study also only used atrial fibrillation when it was the 
most responsible diagnosis. This strategy will miss events where atrial fibrillation is a contributing factor or co-morbid condition. However, as noted in the methods, the most responsible diagnosis is also the most reliably coded in the data base. Finally, no attempt was made to link the seasonality with potential causes such as temperature or ambient air quality.

The magnitude of the observed seasonal effect is small, particularly in comparison to the seasonal effects demonstrated for conditions such as pneumonia [12], asthma [13], and falls [14]. The seasonal effect is not likely of policy, etiologic or clinical relevance. The dramatic increase in trend for hospitalizations is unexplained, and reflects overall increases in hospitalizations during this time period [15]. The analysis by Frost et al did not indicate the existence of trends and as no time plots were provided, it is uncertain whether this phenomenon is restricted to Ontario, or has occurred elsewhere as well. Furthermore, they reported their outcomes as relative risk increases which do not give an indication of the magnitude of effect.

\section{Conclusions}

In conclusion, this study supports a weak, but likely inconsequential seasonal variation in hospital admissions for atrial fibrillation in Ontario.

\section{Competing interests}

None declared.

\section{Authors' contributions}

RU conceived the project and wrote the initial draft of the paper. RM contributed the statistical analysis with the support of MM, RU and EC. All contributed significant intellectual input to the project, contributed to each draft and have read and agree to the contents of the submitted manuscript.

\section{Acknowledgements}

This project was funded by an operating grant (\#MOP57928) from the Canadian Institutes of Health Research. We thank Shari Gruman for her assistance in the preparation of the manuscript. Dr Upshur is supported by a New Investigator Award from the Canadian Institutes of Health Research and a Research Scholar Award from the Department of Family and Community Medicine, University of Toronto.

\section{References}

I. Ceresne L, Upshur RE: Atrial fibrillation in a primary care practice: prevalence and management. BMC Fam Pract 2002, 3:I I.

2. Go AS, Hylek EM, Phillips KA, Chang Y, Henault LE, Selby JV, Singer $D E$ : Prevalence of diagnosed atrial fibrillation in adults: national implications for rhythm management and stroke prevention: the Anticoagulation and Risk Factors in Atrial Fibrillation (ATRIA) Study. JAMA 200I, 285:2370-2375.

3. Viskin S, Golovner M, Malov N, Fish R, Alroy I, Vila Y, Laniado S, Kaplinsky $E$, Roth $A$ : Circadian variation of symptomatic paroxysmal atrial fibrillation. Data from almost 10000 episodes. Eur Heart J 1999, 20:1429-1434.
4. Kupari M, Koskinen P: Seasonal variation in occurrence of acute atrial fibrillation and relation to air temperature and sale of alcohol. Am J Cardiol 1990, 66:1519-1520.

5. Frost L, Johnsen SP, Pedersen L, Husted S, Engholm G, Sorensen HT, Rothman KJ: Seasonal variation in hospital discharge diagnosis of atrial fibrillation: a population-based study. Epidemiology 2002, | 3:21|-2|5.

6. Jenkins G, Watts D: Spectral analysis and its applications San Francisco: Holden Day; 1968.

7. Priestly M: Spectral analysis and time Series New York: Academic Press, Inc; 1981 .

8. Bell W, Hillmer S: Issues involved with the seasonal adjustment of economic time series. Journal of Business \& Economic Statistics 1984, 2:29I-320.

9. SAS/ETS user's guide version 8 Cary, NC: SAS Institute Inc; 1999.

10. Box G, Jenkins G: Time series analysis: forecasting and control San Francisco: Holden-Day; 1976.

II. Moineddin R, Upshur RE, Crighton E, Mamdani M: Autoregression as a means of assessing the strength of seasonality in a time series. Popul Health Metr 2003, I:I0.

12. Upshur RE, Knight K, Goel V: Time-series analysis of the relation between influenza virus and hospital admissions of the elderly in Ontario, Canada, for pneumonia, chronic lung disease, and congestive heart failure. Am J Epidemiol 1999, 149:85-92.

13. Crighton EJ, Mamdani MM, Upshur RE: A population based time series analysis of asthma hospitalisations in Ontario, Canada: 1988 to 2000. BMC Health Serv Res 200I, I:7.

14. Mamdani MM, Upshur RE: Fall-related hospitalizations: what's in season? Can J Public Health 200 I, 92: I I3-II6.

15. Crighton EJ, Moineddin R, Upshur RE, Mamdani M: The seasonality of total hospitalizations in Ontario by age and gender: a time series analysis. Can J Public Health 2003, 94:453-457.

\section{Pre-publication history}

The pre-publication history for this paper can be accessed here:

http://www.biomedcentral.com/1472-6963/4/5/prepub
Publish with Bio Med Central and every scientist can read your work free of charge

"BioMed Central will be the most significant development for disseminating the results of biomedical research in our lifetime. "

Sir Paul Nurse, Cancer Research UK

Your research papers will be:

- available free of charge to the entire biomedical community

- peer reviewed and published immediately upon acceptance

- cited in PubMed and archived on PubMed Central

- yours - you keep the copyright

Submit your manuscript here:

http://www.biomedcentral.com/info/publishing_adv.asp
BioMedcentral 